\title{
Práticas de cuidado de mães de crianças com paralisia cerebral
}

\author{
Sumaya Virgilia Monteiro Braun** \\ Rodolfo Gomes do Nascimento* \\ Samia Marcia Araujo Monteiro Pires *** \\ Katiane da Costa Cunha**** \\ Simone Souza da Costa Silva*****
}

\begin{abstract}
Resumo
Essa pesquisa teve por objetivo investigar as práticas de cuidado de mães de crianças com paralisia cerebral (PC), residentes na cidade de Belém e Região Metropolitana. Para isso foi necessário correlacionar variáveis sociodemográficas com a variável prática de cuidado e suas dimensões. A amostra foi composta por 24 mães de crianças com PC e os instrumentos utilizados foram o Inventário Sociodemográfico, o Sistema de Classificação da Função Motora Grossa, e a Escala de Crenças Parentais e Práticas de Cuidados. Como resultado, foi possível conhecer a influência do comportamento da mãe no desenvolvimento de crianças com paralisia cerebral a partir das características das práticas de cuidado. Em geral, observou-se que as mães apresentam práticas menos efetivas de cuidado, sendo a dimensão Estimulação por objeto (P-EO) a de maior percentual para a quanto às práticas menos efetivas e os Cuidados primários (P-CP) a dimensão de maior percentual de práticas mais efetivas.

Palavras-chave: práticas de criação infantil; paralisia cerebral; parentalidade.
\end{abstract}

\section{Care practices of mothers of children with cerebral palsy}

\begin{abstract}
This research aimed to investigate the care practices of mothers of children with cerebral palsy (CP), living in the city of Belém and the Metropolitan Region. For this, it was necessary to correlate sociodemographic variables with the practical variable of care and its dimensions. The sample consisted of 24 mothers of children with CP and the instruments used were the Sociodemographic Inventory, the Gross Motor Function Classification System, and the Parental Beliefs and Care Practices Scale. As a result, it was possible to know the influence of the mother's behavior on the development of children with cerebral palsy from the characteristics of care practices. In general, it was observed that mothers have less effective care practices, with the dimension Stimulation by object (P-SO) being the highest percentage for the less effective practices and Primary Care (P-PC) the dimension of higher percentage of more effective practices.
\end{abstract}

Key words: children's breeding practices; cerebral palsy; parenting.

* Psicóloga pela PUCRJ e Mestre pelo PPGTPC-UFPA

** Fisioterapeuta pela UNAMA, Doutor e Pós-doutorando pelo PPGTPC-UFPA.

***Psicóloga, Mestre e doutoranda no PPGTPC-UFPA

**** Fisioterapeuta. Doutorado e Pós-Doutorado no PPGTPC-UFPA

***** Psicóloga e mestre pela UFPA. Doutora em psicologia pela UNB. Profa. e diretora adjunta do Núcleo de Teoria e Pesquisa do Comportamento - NTPC-UFPA 


\section{Introdução}

A Paralisia Cerebral (PC) se caracteriza por atraso do desenvolvimento neuropsicomotor. É uma doença crônica gerada por uma lesão no sistema nervoso central ocorrida nos estágios precoces do desenvolvimento. Também conhecida por encefalopatia crônica não progressiva da infância (ECNE), caracteriza-se por ser um distúrbio motor com alterações de movimentação voluntária e do tônus muscular, hipercinesias, distúrbios da aprendizagem e comunicação, podendo provocar limitações ou impedimentos quanto à capacidade de desempenhar as atividades de vida diária (Azevedo \& Barba 2017; Cury \& Brandão, 2011; Morilla, Caldas, Scarpellini \& Santos, 2017).

Diante disto, o diagnóstico da PC pode gerar diversos conflitos emocionais às famílias no confronto com uma realidade que, na maioria dos casos, é desconhecida e desafiadora (Cury \& Brandão, 2011). Os pais que passam por essa experiência terão que viver um longo processo de adaptação em que novos hábitos e rotinas são gerados com vistas a atender as necessidades da criança. Por muitas vezes, essa rotina complexa de cuidados gera sobrecarga ao cuidador, dado o nível de exigência e de preocupações, haja vista a maior dependência da criança, impactando a vida social e a saúde de quem cuida que na maioria das vezes são as mães (Cury \& Brandão, 2011; Hatzman, Maurice-Stam, Heymans, \& Grootenhuis, 2009; Whiting, 2012).

Com relação ao cuidado parental, este se dá a partir da forte conexão entre pais e filhos, sendo constituído por um conjunto de ações contínuas, influenciadas pelas teias de apoios disponíveis e pelo contexto em que se processa (Vieira, Rímoli, Prado \& Chelini, 2009). Acredita-se que, além das práticas de cuidado com a criança, a família necessita de apoio que contribua para a satisfação das necessidades de interdependência do indivíduo. Este apoio, por sua vez, pode advir de elementos que tecem a rede na qual a família busca a ajuda para o cuidado da criança (Milbrath, Siqueira, Amestoy \& Trindade, 2011; Sluzki, 2010).

A partir da compreensão de que o comportamento dos pais com seus filhos está, também, associado às características da criança, algumas evidências apontam que a parentalidade em famílias de crianças com deficiência constitui uma prática que se diferencia daquelas observadas em contextos familiares com crianças típicas. Desta forma, dependendo das características da deficiência, os pais desempenharão a parentalidade de forma única, como por exemplo, naquelas famílias constituídas por uma criança com PC (Cunha, Ramos, Silva \& Pontes, 2017).
Os cuidados dispensados pelos pais podem ser considerados tarefas de desenvolvimento universais. No entanto, a forma como os pais os exerce pode variar de acordo com as configurações contextuais e culturais nas quais a família está inserida (Keller, Abels, Borke, Lamm, Su, Wang \& Lo, 2007).

Em 2007, Heidi Keller criou um modelo denominado "Modelo de Investimento Parental" com o propósito de compreender a parentalidade humana. Definidos como um conjunto de comportamentos biologicamente preparados e ativados pelas demandas ambientais, esses sistemas têm por objetivo promover a proximidade e o conforto quando a criança está em risco real ou potencial (Martins, Vieira, Moura \& Macarini, 2011).

Diante disto, os contextos, segundo a pesquisadora se associam à ideias específicas de responsabilidade sobre o desenvolvimento infantil. E estas, por sua vez, tomam um sentido mais concreto por meio de seis sistemas que representam os cuidados parentais culturalmente compartilhados e abrangem comportamentos de promoção de conforto em meio a situações de risco real ou potencial para a criança. Estes estão divididos em cuidado primário (representa os primeiros cuidados da infância), contato corporal (consiste em tocar e carregar a criança de forma próxima ao corpo do cuidador), estimulação corporal (visa à comunicação corporal por meio do toque e movimentos motores no corpo da criança), estimulação por objetos (consiste na utilização de brinquedos durante a interação entre cuidador e criança), contato face a face (mútuo contato visual entre cuidador e criança e o frequente uso da linguagem) e envelope narrativo (consiste na utilização da linguagem pelos cuidadores) (Keller, 2007; Seidl-de-Moura, Pessôa, Ramos, Mendes, Fioravanti-Bastos \& Dias, 2014).

Os estudos sobre famílias de crianças com deficiência, em particular, famílias de pessoas com PC, tem mostrado particular interesse em compreender os aspectos relativos à sobrecarga, estresse, rede de apoio etc. (Cunha, 2016; Pires, 2017), pouco sendo conhecido sobre as práticas parentais adotadas por estes cuidadores no contexto familiar. O fato é que existe uma ampla literatura sobre práticas parentais que embora se sustente em modelos teóricos robustos se propõe a refletir sobre as práticas parentais em contextos típicos de desenvolvimento, tendo sido pouco usada para explorar sistemas familiares que se afastam dos padrões considerados normais para a sociedade moderna.

Refletir sobre a eficiência das práticas de cuidados às crianças com essas especificidades depende, em grande 
parte, da atenção às necessidades das mães, as quais são, em maioria, as cuidadoras principais e vivenciam rotinas exaustivas (Afonso, Cunha, Pontes \& Silva, 2019). Considerando, portando a importância de se conhecer as características desse cuidado, a presente pesquisa teve como objetivo investigar as práticas de cuidados de mães de crianças com paralisia cerebral.

\section{Método}

\section{Cenário do estudo}

A pesquisa foi realizada em salas previamente liberadas pelos responsáveis das seguintes instituições: Hospital Bettina Ferro de Souza - Universidade Federal do Pará - UFPA; Núcleo de Desenvolvimento em Tecnologia Assistiva e Acessibilidade - NEDETA - Universidade Estadual do Pará - UEPA; e na Unidade de Ensino e Assistência em Fisioterapia e Terapia Ocupacional (UEAFTO/ UEPA). A escolha desses cenários para a coleta de dados decorreu pelo fato de serem serviços de referência no atendimento de crianças com deficiência e pela disponibilidade de atendimento ambulatorial.

\section{Participantes do estudo}

Participaram desta pesquisa 24 mães de crianças com paralisia cerebral (com idades de 0 a 5 anos) atendidos em serviços de referência do estado do Pará. As participantes foram selecionadas pelo critério de acessibilidade nos locais de coleta e por conveniência do pesquisador, devido à facilidade de acesso e trânsito dentro das instituições. Este tipo de amostra é considerado não probabilística, pois a seleção dos participantes não é orientada por critérios estatísticos, mas por estarem acessíveis ao pesquisador.

Os critérios de inclusão para participação na pesquisa foram: ser mãe da criança com idade de 0 a 5 anos com paralisia cerebral; estar em atendimento nos locais de coleta; residir na região metropolitana de Belém e residir na mesma casa da criança.

Do total, $13(19,99 \%)$ se declararam casadas ou em união estável, $11(16,93 \%)$ tinham escolaridade inferior a 11 anos, e $13(20,00 \%)$ tinham de 11 ou mais anos de escola formal. Quanto à religião, $15(23,07 \%)$ mães assumiram serem católicas, e $6(9,22 \%)$ evangélicas. As crianças deste grupo se caracterizam em números semelhantes para masculino e feminino, a maioria está na faixa etária de 4 a 5 anos, e não se encontram inseridas no ensino formal. Na GMFCS, 7 (29,17\%) crianças se encontravam no nível IV.

\section{Instrumentos de coleta de dados}

Foram utilizados três instrumentos na coleta de dados: Inventário sociodemográfico (ISD); Sistema de Classificação da Função Motora Grossa (GMFCS) e Escala de Crenças Parentais e Práticas de Cuidados (E-CPPC).

Sobre o ISD, este foi elaborado pelo Laboratório de Ecologia do desenvolvimento - LED, é composto por cinco seções: 1: Informações sobre atendimento; 2: Composição familiar da criança; 3: Dados sobre os cuidadores principais; 4: Características socioeconômicas; e 5: Dados das crianças. Esta última seção foi dividida em duas subseções. A: Dados de crianças com PC; e B: Dados de crianças com desenvolvimento típico.

Quanto ao GMFCS, é uma escala de medida da função motora grossa de criança com paralisia cerebral (PC), que avalia o desempenho motor infantil de acordo com a idade. Esta escala apresenta-se organizada em cinco níveis de classificação. A criança que se apresenta no Nível I - anda sem limitações; no Nível II - anda com limitações; no Nível III - anda utilizando um dispositivo manual de mobilidade; no Nível IV - apresenta automobilidade com limitações, pode utilizar mobilidade motorizada; no Nível V - transportado em uma cadeira de rodas manual (Palisano, Rosenbaum, Bartlett \& Livingston, 2008).

Com relação à E-CPPC, foi utilizada a versão construída e validada para o Brasil por Martins, Vieira, Moura \& Macarini, 2011, esta tem por objetivo identificar as crenças e práticas de cuidados parentais construídas a partir do modelo teórico de Keller (2007). Organizada em cinco dimensões baseada no sistema de comportamento parental descrito por Keller (2007), utilizado por adultos na interação e cuidados com as crianças. São eles: cuidados primários, contato corporal, estimulação corporal, estimulação por objetos, contato face a face. Esta escala foi utilizada em duas amostras com 250 e 600 mães, que apresentaram Alfas de Cronbach variando entre 0,68 e 0,83 . Assim, estes autores sugeriram que tal medida reuniu evidências de validade fatorial e confiabilidade.

\section{Procedimentos de coleta de dados}

A coleta de dados foi realizada em 2016 por pesquisadores vinculados ao projeto de pesquisa. Neste primeiro momento, as mães e as crianças foram localizadas e identificadas por meio dos prontuários clínicos examinados. Após o levantamento do número de crianças com PC que se enquadravam nos critérios escolhidos, os participantes eram abordados e entrevistados na sala de espera nos dias em que as crianças possuíam atendimentos agendados. 
Neste encontro, eram feitos esclarecimento da pesquisa e realizado o convite para participar.

Ressalta-se que a coleta foi realizada após autorização expedida pelos respectivos diretores dos locais de coleta. Após esclarecimentos sobre a pesquisa, as mães eram convidadas a participar da mesma. Os instrumentos aplicados seguiram a seguinte ordem: ISD; GMFCS (no grupo PC) e E-CPPC. A aplicação da escala GMFCS foi realizada por fisioterapeutas que faziam parte da equipe de pesquisa. As mães foram questionadas sobre o desempenho habitual da criança em casa, na escola e nos espaços comunitários (Cunha, 2016).

\section{Procedimentos de análise e interpretação dos dados}

Após a realização da coleta, os dados foram inicialmente inseridos em planilhas construídas no programa Excel 2010, sendo categorizados e tratados. Posteriormente foram analisados no aplicativo Statistical Package for the Social Sciences - SPSS, versão20.0@ para o Windows e no aplicativo Statistica versão 6.0, onde se gerou análises estatísticas descritivas dos grupos de mães e das crianças. A técnica estatística utilizada nesta pesquisa foi a Análise de Correspondência (ANCOR) e o teste qui-quadrado.

Para realizar a Análise de Correspondência todas as variáveis foram divididas em dois ou três grupos/categorias. As variáveis referentes às práticas (P-CP; P-CC; P-EC; P-EO; P-EF), foram categorizadas em Baixo(a) e Alto(a) a partir do valor do terceiro quartil (Q3) dos valores observados (Bussab \& Morettin, 2017), isto é, variáveis com valores abaixo de Q3 foram categorizadas como menos efetivas e, consequentemente, variáveis com valores acima de Q3 foram categorizadas mais efetivas. Em todos os testes fixou-se $\alpha=5 \%(p \leq 0,05)$ para rejeição da hipótese nula.

\section{Considerações e aspectos éticos}

A pesquisa foi aprovada pelo Comitê de Ética do Instituto Evandro Chagas, com parecer de $\mathrm{n}^{\circ}$ 1.758.751, na data de 03/10/2016. A participação dos cuidadores ocorreu após assinatura do Termo de Consentimento Livre e Esclarecido - TCLE, mediante informações prévias a respeito do conteúdo do mesmo. Foram apresentados os propósitos da pesquisa, conforme previsto na Resolução no 510, de 07 de abril de 2016, do Conselho Nacional de Saúde/Ministério da Saúde, que dispõe sobre as normas de pesquisas envolvendo seres humanos. Garantindo, assim, aos participantes confidencialidade das informações e o direito de acesso aos resultados.

\section{Resultados}

A Tabela 1 mostra $O$ percentual de famílias de crianças com paralisia cerebral de acordo com a categorização práticas mais efetivas e menos efetivas de cuidado, bem como suas dimensões: cuidado primário, contato corporal, estimulação corporal, estimulação por objeto e face a face. A partir dos dados gerais, observa-se que as mães apresentam práticas menos efetivas de cuidado $(75,00 \%)$. No detalhe, verificou-se o maior percentual para a dimensão $\mathrm{P}-\mathrm{EO}$ quanto às práticas menos efetivas de cuidado $(75,00 \%)$ e a dimensão P-CP registrou o maior percentual de práticas mais efetivas $(66,67 \%)$.

Tabela 1. Percentual geral das práticas de cuidados das mães de crianças PC e suas dimensões, em função da categorização (menos e mais efetivas). Belém-PA, 2014 a 2017. $(n=65)$

\begin{tabular}{llllllll}
\hline \multicolumn{7}{c}{ PRÁTICAS } \\
\hline Grupo & Categoria & Geral & CP & CC & EC & EO & FF \\
\hline & Baixa & 75,00 & 33,33 & 58,33 & 41,67 & 75,00 & 54,17 \\
\multirow{2}{*}{ Mães PC } & Alta & 25,00 & 66,67 & 41,67 & 58,33 & 25,00 & 45,83 \\
& Total & 100 & 100 & 100 & 100 & 100 & 100 \\
\hline
\end{tabular}

Fonte: pesquisa de campo

Nota: PC: paralisia cerebral / CP - cuidado primário; CC - contato corporal; EC - estimulação corporal; EO - Estimulação por objeto; FF - face a face.

As associações entre as variáveis sociodemográficas e as cinco dimensões das práticas de cuidado. Considerando a dimensão cuidado primário (P-CP) observou-se que as práticas menos efetivas de cuidado estavam associadas fortemente com a mães de idade entre $23 \mathrm{e}$ 30 anos, casada ou em união estável, escolaridade de 11 ou mais anos, ser ateia, estar inserida no serviço formal com renda mensal familiar de até 1 salário-mínimo ou de 2 a 3 salários-mínimos, e ter filho do sexo masculino. Por outro lado, as práticas mais efetivas de cuidado estavam significativamente associadas às mães com idade menor que 23 anos e maior que 30 anos, ser solteira, escolaridade menor que 11 anos, evangélica, ocupação do lar, estudante ou desempregada, com Renda Média Familiar (RMF) de 1 a 2 salários-mínimos, e ter filho do sexo feminino.

Sobre a dimensão contato corporal (P-CC), as práticas maternas de cuidado menos efetivas estavam associadas às mães com idade entre 23 e 30 anos e maior que 30 anos, solteira, evangélica, ocupação formal com RMF de até 1 salário-mínimo e de 2 a 3 salários-mínimos, e filhos 
do sexo masculino. Por outro lado, as práticas mais efetivas foram mais observadas nas mães com idade menor de 23 anos, casadas, ateias, ocupação do lar, estudantes ou desempregadas, com RMF de 1 a 2 salários-mínimos, e filho do sexo feminino.

Com relação à dimensão estimulação corporal (P$\mathrm{EC})$, as práticas maternas de cuidado menos efetivas estavam significativamente relacionadas às mães com idade entre 23 e 30 anos, escolaridade de 11 ou mais anos, ser católica, ocupação do lar, estudante ou desempregada, com RMF de até 1 salário-mínimo, e filho do sexo masculino. Quanto à alta prática de cuidado, foi identificado em mães com idade menor que 23 anos e maior que 30 anos, ter menos de 11 anos de escolaridade, ser evangélica ou ateia, inserida no serviço formal com RMF de 2 a 3 salários-mínimos, e filho do sexo feminino.

Acerca da dimensão estimulação por objeto (P-EO), as práticas menos efetivas de cuidado estavam fortemente associadas às mães com idade entre 23 e 30 anos, solteiras, escolaridade de 11 ou mais anos, católicas, ocupação do lar, estudantes ou desempregadas, com RMF de 1 a 2 salários-mínimos, e filhos do sexo feminino com idade maior de 3 anos. Enquanto, as práticas mais efetivas de cuidado estavam associadas a mães com idade menor que 23 anos e maior que 30 anos, casadas ou em união estável, de nível escolar menor que 11 anos, evangélicas ou ateias inseridas no serviço formal com RMF de 2 a 3 salários-mínimos, e filhos do sexo masculino de idade de 1 a 3 anos.

Considerando a dimensão face a face (P-FF), as práticas menos efetivas de cuidado estavam associadas às mães com idade entre 23 e 30 anos e maior que 30 anos, casada ou em união estável, escolaridade de 11 ou mais anos, católicas ou ateias, ocupação do lar, estudantes ou desempregadas, com RMF de 1 a 2 salários-mínimos. Por outro lado, as práticas mais efetivas de cuidado para esta dimensão estavam fortemente associadas às mães de idade menor que 23 anos, solteiras, escolaridade menor que 11 anos, evangélicas, inseridas no serviço formal com RMF de até 1 salário-mínimo e de 2 a 3 salários-mínimos.

\section{Discussão}

Diante das associações efetivadas entre variáveis sociodemográficas e práticas de cuidados de mães de crianças com Paralisia Cerebral, a idade chama atenção. As mães mais jovens apresentam maior disponibilidade na sua rotina diária, podendo-se entender que elas não estejam inseridas em um serviço formal, e devido a esta característica, conseguem exercer sua parentalidade de forma mais contínua. Por outro lado, mães com idade maior de 30 anos, apresentam maior experiência, maior conhecimento e, algumas vezes, maior estrutura emocional e financeira, o que poderia vir a favorecer a busca de técnicas e instrumentos para estimular o desenvolvimento do filho com PC. Este dado corrobora com os estudos que apontam como fator protetivo a idade elevada dos pais, destacando que a consciência sobre as funções parentais está diretamente relacionada com a experiência de vida (Rothstein \& Beltrame, 2013; Azevedo \& Barba, 2017).

$\mathrm{Na}$ correlação envolvendo as variáveis situação civil e religião, foram evidenciadas práticas efetivas de cuidado em mães de crianças com PC solteiras e evangélicas nas dimensões de cuidado primário e face a face, e para mães casadas ou em união estável nas dimensões de contato corporal e estimulação por objeto. Estudos confirmam que a situação civil das mães é bastante significativa na condução de sua parentalidade. Para alguns autores, ter um parceiro no contexto família de criança com PC passa a ser um fator protetivo para essa mãe, ou seja, a presença dos dois cônjuges, se configura como fator de proteção ao desenvolvimento infantil, visto a possibilidade de um maior compartilhamento de cuidados e aumento de apoio para o cuidador principal (Morilla, Caldas, Scarpellini \& Santos, 2017).

Outra variável importante a ser destacada diz respeito ao nível de escolaridade das mães de crianças com PC onde, segundo a literatura, esta variável também se configura como fator protetivo (Afonso, 2016; Azevedo \& Barba, 2017). Os dados obtidos neste estudo apontam que as práticas mais efetivas no grupo de mães PC estão relacionadas a mães com baixa escolaridade, que apresentam resultados significativos para as dimensões de cuidado primário, estimulação corporal, por objeto e por face a face. Estes resultados não corroboram com os descritos na literatura, o que permite supor que mais do que os anos de escolaridade é preciso considerar a qualidade da educação acessada pelas participantes.

Sobre a variável ocupação, a literatura aponta as mães como cuidadoras principais, e que muitas vezes não conseguem se inserir no mercado de trabalho formal em decorrência das demandas do filho (Afonso, 2016; Cunha, 2016; Souza, 2017; Reis, Alves, Barbosa, Lomba \& Braga, 2017). Os resultados obtidos neste trabalho corroboram com a literatura em que se encontrou práticas mais efetivas de cuidado relacionadas a mães do lar, estudantes ou desempregadas, com RMF de 1 a 2 SM nas dimensões cuidado primário e contato corporal. Este dado possibilita levantar a hipótese que devido à possibilidade de um 


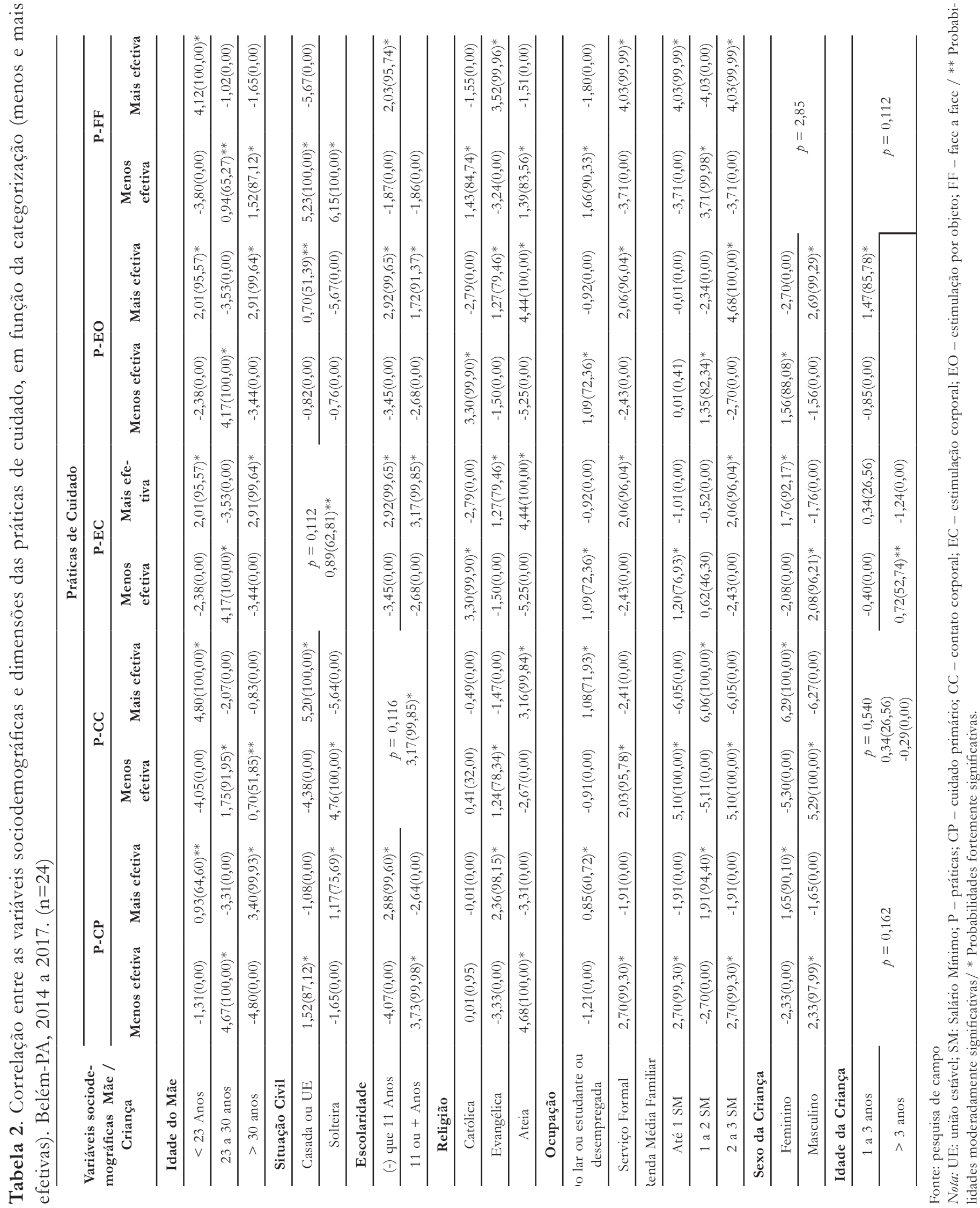


convívio maior nas atividades do dia a dia, estas mães se apresentam com maior disponibilidade para os cuidados primários e contato corporal.

Com relação aos dados envolvendo as práticas menos efetivas destaca-se as seguintes discussões. Considerando as dimensões cuidado primário, estimulação corporal, estimulação por objeto e face a face, estas estavam associadas à alta escolaridade das mães. Este dado confronta evidência já descrita na literatura, a qual relaciona tais práticas com a baixa escolaridade do cuidador por implicar numa menor compreensão acerca da doença do filho, sendo este um dificultador para o desenvolvimento da parentalidade (Souza, 2017).

A despeito das associações entre práticas menos efetivas de cuidado e os dados envolvendo renda, muitas das participantes deste estudo recebiam o benefício social por meio do Benefício de Prestação Continuada - BPC, que é um benefício assistencial à pessoa com deficiência ou à Bolsa Família, configurando a baixa renda destas famílias. Lima (2016) em sua pesquisa sinalizou que a vulnerabilidade social das famílias de pessoas com PC, tem sido associada a uma pior qualidade de vida e um maior risco ao desenvolvimento saudável tanto da mãe quanto da criança. Portanto, pode-se pensar que estes fatores favorecem o aumento das dificuldades para o exercício da parentalidade.

\section{Considerações finais}

Esta pesquisa forneceu possibilidades de ampliar o conhecimento acerca das práticas parentais de mães de crianças com PC, identificando a influência do comportamento das mães no desenvolvimento de crianças com paralisia cerebral, e suas possíveis correlações com as variáveis sociodemográficas. Portanto, conclui-se que as práticas mais e menos efetivas de cuidado materno apresentaram configurações heterogêneas em função dos domínios analisados.

Sabe-se que as características culturais de cada contexto familiar, influenciam diretamente nas percepções que as mães desenvolvem para o exercício de suas práticas parentais. Pensando no papel dos profissionais que atuam no atendimento das crianças e no acompanhamento das mães, esta pesquisa apresenta-se como relevante, pois sinaliza as implicações positivas e negativas do cuidado parental e dá visibilidade à necessidade do amparo e escuta acolhedora durante os atendimentos das mães.

Vale ressaltar que este estudo teve o limitador de avaliar as práticas de cuidado apenas em um dos membros da família, as mães. Entretanto, a vivência de um membro com PC é de toda família. Sendo assim, sugere-se que estudos envolvendo outros membros da família sejam realizados, além da inclusão de dados qualitativos por meio de entrevistas para um aprofundamento sobre a percepção e as narrativas das mães e familiares.

\section{Referências}

Afonso, T (2016). Práticas de cuidado, redes de apoio e satisfação social de cuidadores primários de crianças com paralisia cerebral. Tese de Doutorado não publicada, Curso de Pós-Graduação em Teoria e Pesquisa do Comportamento, Universidade Federal do Pará.

Afonso, T., Cunha, K. D. C., Pontes, F. A. R., \& Silva, S. S. D. C. (2019). Percepção materna acerca dos cuidados na paralisia cerebral: a importância do apoio. Pensando famílias, 23(2), 177-190.

Azevedo, T., \& Barba, P. C. D. (2017). Avaliação da estimulação e apoio no ambiente familiar oferecido à criança com paralisia cerebral. Revista De Terapia Ocupacional Da Universidade De São Paulo, 28(2), 198-205. https://doi.org/10.11606/issn.2238-6149.v28i2p198-205

Bussab, W. O., Morettin, P. A. (2017). Estatística Básica. 9.ed., Saraiva.

Cunha, K. C. (2016) Estresse e resiliência em pais de crianças com paralisia cerebral. Tese de Doutorado apresentada ao Programa de Pós-graduação em Teoria e Pesquisa do Comportamento, Núcleo de Teoria e Pesquisa do Comportamento, Universidade Federal do Pará.

Cunha, K. C., Ramos, M. F. H., Silva, S. S. C., \& Pontes, F. A. R. (2017). Estresse parental e paralisia cerebral. Psicologia, Saúde \& Doenças, 18(2), 433-450. https://dx.doi.org/10.15309/17psd180212

Cury, V. C. R., \& Brandão, M. (2011). Reabilitação em paralisia cerebral. Rio de Janeiro: MedBook.

Hatzmann, J., Maurice-Stam, H., Heymans, H. A. S., \& Grootenhuis, M. A. (2009). A predictive model of health-related quality of life of parents of chronically ill children: The importance of care-dependency of their child and their support system. Health and Quality of Life Outcomes, 7, 72-81. doi:10.1186/1477-7525-7-72

Keller, H. (2007). Cultures of Infancy. New Jersey: Lawrence Erlbaum Associates.

Keller, H., Abels, M., Borke, J., Lamm, B., Su, Y., Wang, Y., \& Lo, W. (2007). Socialization environments of Chinese and Euro-American middle-class babies: Parenting behaviors, verbal discourses and ethnotheories. International Journal of Behavioral Development, 31(3), 210-217.

Lima, M. B. S. (2016). Estresse Parental de cuidadores de crianças com paralisia cerebral no Estado do Pará. Tese de Doutorado apresentada ao Programa de Pós-graduação em Teoria e Pesquisa do Comportamento, Núcleo de Teoria e Pesquisa do Comportamento, Universidade Federal do Pará.

Martins, G.D.F., Vieira, M. L., Moura, M. L.S.D., \& Macarini, S.M. (2011). Crenças e práticas de cuidado entre mães residentes em capitais e pequenas cidades Brasileiras. Psicologia: Reflexão e Crítica, 24(4).

Milbrath, V. M., Siqueira, H. C. H., Amestoy, S. C., \& Trindade, L. L. (2011). Redes de apoyo utilizadas por la familia cuando el niño nace com necessidades especiales. Evidentia, 8(36).

Morilla, C.M., Caldas, C.A.C.T., Scarpellini, A.C.A.V., Santos, P.L. (2017) Family resources and promotion of development of children with cerebral palsy. Journal of Human Growth and Development, 27(2): 166-174. doi: http:// dx.doi.org/10.7322/jhgd.122707

Palisano, R., Rosenbaum, P., Bartlett, D., \& Livingston, M. (2008). Content validity of the expanded and revised Gross Motor Function Classification System. Dev Med Child Neurol, 50(10), 744-750. doi: 10.1111/j.1469$-8749.2008 .03089 . x$

Pires, S. M. A. M. (2017). Redes pessoais de cuidadores de crianças com paralisia cerebral e desenvolvimento típico. Dissertação de Mestrado. Programa de Pós-Graduação em Teoria e Pesquisa do Comportamento. Belém: Universidade Federal do Pará.

Reis, K.M.N., Alves, G.V., Barbosa, T.A., Lomba, G.O., Braga, P.P. (2017). A vivência da família no cuidado domiciliar à criança com necessidades especiais de saúde. Revista Ciencia y Enfermeria, XXIII (1), 45-55.

Rothstein J.R., Beltrame T.S. (2013). Características motoras e biopsicosso- 


\section{SUMAYA VIRGILIA MONTEIRO BRAUN et al.}

ciais de crianças com paralisia cerebral. Revista Brasileira de Ciência e Movimento, 21(3), 118-126. http://dx.doi.org/10.18511/0103-1716/rbcm. v21n3p118-126

Sluzki, C. E. (2010). Personal social networks and health: conceptual and clinical implications of their reciprocal impact. Families, Systems, \& Health, $28(1), 1$.

Seidl-de-Moura, M. L., Pessôa, L. F., Ramos, D. D. O., Mendes, D. M. L. F., Fioravanti-Bastos, A. C. M., \& Dias, L. B. T. (2014). Beliefs of Mothers, Nannies, Grandmothers and Daycare Providers Concerning Childcare1. Paidéia (Ribeirão Preto), 24(59), 341-349.

Souza, P. B. M. (2017). Coparentalidade e Auto-eficácia de cuidadores de crianças com paralisia cerebral. Tese de doutorado apresentada ao Programa de Pós-Graduação em Teoria e Pesquisa do Comportamento, Núcleo de Teoria e Pesquisa do Comportamento, Universidade Federal do Pará.
Vieira, M. L., Rímoli, A. O., Prado, A. B., \& Chelini, M. O. (2009). Cuidado e responsividade parentais: Uma análise a partir da Teoria da História de Vida e da Teoria do Investimento Parental. Psicologia evolucionista, 86-95.

Whiting, M. (2012). Impact, meaning and need for help and support: The experience of parents caring for children with disabilities, life-limiting/ life-threatening illness or technology dependence. Journal of Child Health Care, 17(1), 92-108. doi:10.1177/1367493512447089

Submetido em: 20-7-2020

Aceito em: 8-3-2021 